\title{
Computed Tomography-based Morphological Differences between Histologic Subtypes of Periampullary Ductal Adenocarcinoma
}

\author{
Nesrin Gündüz ${ }^{1}$, Fatih Buyuker ${ }^{2}$, Hatice Seneldir ${ }^{3}$, Gulcin Durukan ${ }^{1}$, Orhan Alimoglu ${ }^{2}$ and Adnan Kabaalioglu ${ }^{1}$ \\ ${ }^{1}$ Department of Radiology, Faculty of Medicine, Istanbul Medeniyet University, Göztepe Training and Research Hospital, Turkey \\ ${ }^{2}$ Department of General Surgery, Faculty of Medicine, Istanbul Medeniyet University, Göztepe Training and Research Hospital, Turkey \\ ${ }^{3}$ Department of Pathology, Faculty of Medicine, Istanbul Medeniyet University, Göztepe Training and Research Hospital, Turkey
}

\begin{abstract}
Objective: To evaluate the computed tomography (CT)-based differences between pancreaticobiliary (PBST) and intestinal (IST) subtypes of periampullary pancreatic ductal adenocarcinomas (PDAC).

Study Design: Analytical study.

Place and Duration of Study: Faculty of Medicine, Istanbul Medeniyet University, Göztepe Training and Research Hospital, Turkey between 2015 and 2018.

Methodology: Overall 24 periampullary PDAC cases, in whom histomorphologic evaluation and CDx2 expression were used to discriminate between PBST and IST, were included. The lesion morphology (infiltrative versus nodular), common bile and main pancreatic ducts' dilation, tumor grade, enhancement pattern, pancreaticoduodenal groove, pancreaticoduodenal artery and lymphatic involvement were evaluated by CT.

Results: Overall 24 PDAC cases [median age 67.5 (60.5-76.5) years] were enrolled. Histopathology revealed 9 (25\%) IST and 18 (75\%) PBST. The age [72.5 (69-81) versus 63 (57.75-75.5) years, respectively, $p=0.204]$ and gender [3 (50\%) versus $12(66.7 \%)$ males, respectively, $p=0.635$ ] and the prevalence of all CT characteristics were similar between groups ( $p>0.05$ for all) except for lesion morphology. Infiltrative morphology was more frequent in PBST than IST [14 (77.8\%) versus 1 (16.7\%), respectively, p=0.015]. Multiple variable logistic regression analysis revealed infiltrative morphology as the only independent CT predictor of PBST [OR: 14.9, 95\% Cl: 1.2-186), $p=0.036$ ]. The interrater reproducibility for lesion morphology was moderate (Cohen's Kappa: 0.55, $p<0.007)$.

Conclusion: Infiltrative appearance is associated with PBST; whereas, nodular appearance more likely predicts IST. The potential role of CT lesion morphology on guiding appropriate chemotherapy in cases with no chance for surgery or biopsy requires addressing.
\end{abstract}

Key Words: Intestinal differentiation, Pancreatobiliary differentiation, Periampullary adenocarcinoma.

How to cite this article: Gündüz N, Buyuker F, Seneldir H, Durukan G, Alimoglu O, Kabaalioglu A. Computed Tomography-based Morphological Differences between Histologic Subtypes of Periampullary Ductal Adenocarcinoma. J Coll Physicians Surg Pak 2021; 31(08):959-964.

\section{INTRODUCTION}

The periampullary region includes four anatomical structures including pancreatic duct (periampullary portion in the head of pancreas), distal bile duct, ampulla of Vater and duodenum. ${ }^{1}$ The origin of the tumor does not necessarily impact the selection of treatment strategy. ${ }^{2}$ Indeed, pancreatoduodenectomy with an intent of $\mathrm{R} 0$ resection is the only curative option in most cases with resectable tumors. ${ }^{2}$

Correspondence to: Dr. Nesrin Gündüz, Department of Radiology, Faculty of Medicine, Istanbul Medeniyet UniversityGöztepe Training and Research Hospital, Turkey E-mail: gunduz.nesrin@gmail.com

Received: September 06, 2020; Revised: December 18, 2020; Accepted: January 06, 2021

DOI: https://doi.org/10.29271/jcpsp.2021.08.959
Pancreatic head (i.e. pancreatic duct within the periampullary region) cancers have the worst course when compared with tumors of other locations of periampullary region. ${ }^{2}$ Histological differentiation of the tumor cells has emerged as an important underlying biological mechanism leading to divergent behaviour of periampullary cancers originating from different locations. ${ }^{3}$ Two main differentiation subtypes are the intestinal subtype (IST) and the pancreatobiliary subtype (PBST).A longer survival with IST was first reported for ampullary adenocarcinomas. ${ }^{4,5}$ Subsequently, a poor prognosis of PBST was also reported in duodenal and distal bile duct cancers. ${ }^{6}$ Intestinal differentiation has recently been recognised in pancreatic ductal adenocarcinomas (PDAC) of periampullary origin. ${ }^{7,8} \mathrm{~A}$ longer survival was also associated with $C D \times 2$ positive (a marker of intestinal differentiation) periampullary cancers. ${ }^{9}$ The impact of histological subtype not only on survival and prognosis, but also on response to chemotherapy has also been reported in patients with periampullary tumors. ${ }^{10}$ 
Contrast enhanced dynamic computed tomography (CT) provides invaluable information regarding the diagnosis, staging and resectability of the tumour. ${ }^{11}$ Both MRI and CT have successfully differentiated IST and PBST in patients with ampullary cancer. ${ }^{12}$

Previous imaging studies comprised ampullary, distal bile duct, and duodenal periampullary cancers in terms of distinction between IST and PBST. To the best of authors' knowledge, PDAC has not been previously represented in imaging studies in this regard. It was hypothesised that presurgical imaging guided differentiation between IST and PBST in periampullary PDAC would significantly contribute to the evaluation and management of subjects with metastasis and/or those who are not candidates for surgery or invasive sampling. Hence, the aim of this study was to comparethe differential CT and clinical characteristics between IST and PBST in a cohort of pure periampullary PDAC.

\section{METHODOLOGY}

This study comprised patients with pathologically confirmed PDAC of periampullary region, either after surgery or ERCPguided biopsy at Faculty of Medicine, Istanbul Medeniyet University, Göztepe Training and Research Hospital, Turkey between May 2015 and July 2018. Contrast enhanced dynamic CT images were reloaded from the picture archiving communication system of this Institute. Overall, 52 patients with periampullary tumors were initially scanned. For the purpose of this study, only 24 cases with a definitive histopathological diagnosis of PDAC (18 after pancreatoduodenectomy and six after biopsy) were included. The remaining 28 patients were excluded since 14 had non-PDAC periampullary cancers (ampulla or common bile duct tumors), seven had pancreatic head cancer of non-periampullary origin, two had pancreatic head invasion of colon tumor, two had neuroendocrine tumor, one had signet ring cell carcinoma, one had clear cell carcinoma, and one had no malignancy.

CT was performed in all cases before surgery or biopsy. Two radiologists, who were blinded to the clinical and histopathological data, reviewed all the CT images on separate sessions. An additional session was performed for eliminating the discrepancies between readers by consensus. Institutional Ethics Committee approval was obtained (No. 2018/0015). Patients' demographic and clinical features, the date of CT imaging, predefined CT characteristics, and histopathological findings were recorded into a database

The hematoxylin- and eosin-stained slides of all cases were reviewed by an experienced pathologist, blinded to the imaging data. Immunohistochemical analysis (CDx2 expression) was performed in addition to histomorphological assessment. For CDx2, a strong nuclear staining of more than $10 \%$ of tumor cells was considered as CDx2 positivity, which supported IST. Since low number of patients in each of four T stages would not allow for meaningful statistical comparisons, the $\mathrm{T} 1$ and $\mathrm{T} 2$ were merged to form a T-Low group, and T3 and T4 formed a T-High group.

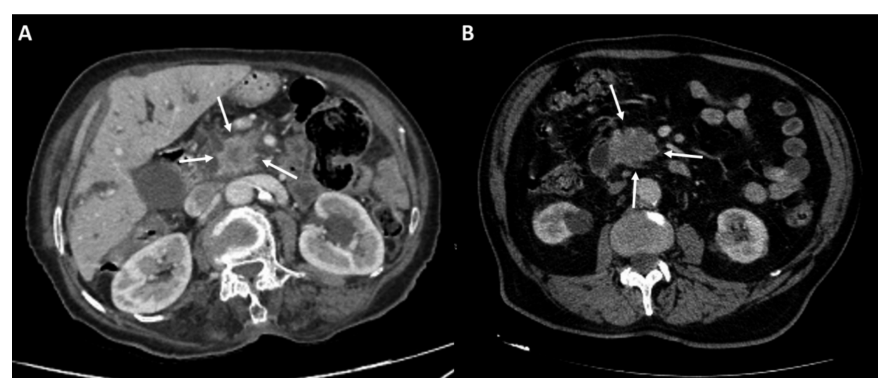

Figure 1: (A) Infiltrative periampullary PDACs with irregularborders. Infiltrative pattern (white arrows) due to prominent spicules. (B) Another case (white arrows) with relatively smoother surface but apparent indentations making it infiltrative.

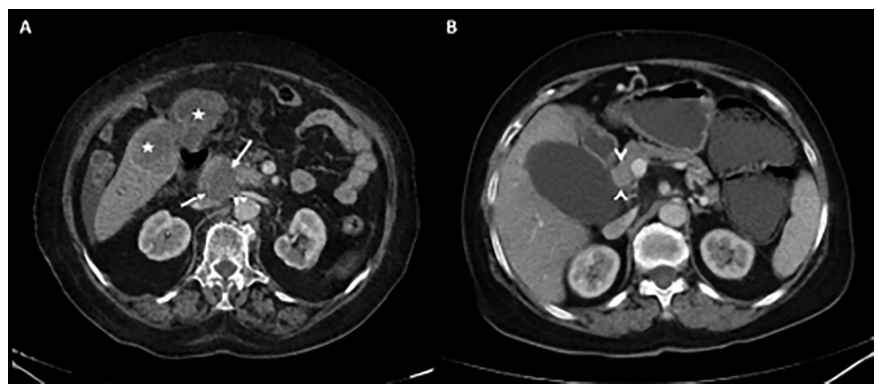

Figure 2: (A) Hypoenhanced nodular periampullary adenocarcinoma (arrows) along with metastases in the liver (asterisks). (B) Hyperenhanced nodular periampullary adenocarcinoma (arrowheads).

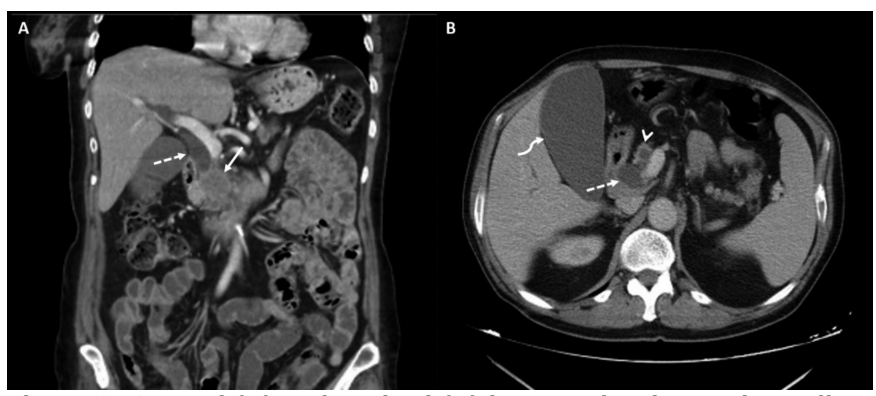

Figure 3: Coronal (A) and sagittal (B) images showing periampullary adenocarcinoma (straight arrow) along with dilated common bile duct (dashed arrow) and main pancreatic duct (arrowhead) and distended gallbladder (curved arrow).

The scanner was a 128-slice MDCT (GE Healthcare Optima CT660, USA). The scanning protocol included pre-contrast and dynamic contrast enhanced [ $1.5 \mathrm{~mL} / \mathrm{Kg}$ of iopromide (Ultravist 370; Schering, Berlin, Germany)] imaging. Using bolus tracking, the late arterial phase at 35th second, and portal venous phase at 60 th second were obtained. The images were reconstructed with a slice thickness of 2.5-3.0 mm and interval of 1.5-2 mm. The predefined CT characteristics were based on the study by Ivanovic et al. with small modifications. ${ }^{13}$

Lesion morphology was defined infiltrative (Figure 1); masses with small protrusions causing irregular or spicular margins; or nodular (Figure 2), well circumscribed mass with smooth borders. Mixed cases were considered as infiltrative.

Common bile duct (CBD) cut-off was categorised into abrupt and blunt termination of common bile duct. A CBD maximum diameter exceeding $6 \mathrm{~mm}$ was accepted as dilated in young (<60 years) (Figure $3 A$ ). 


\begin{tabular}{|c|c|c|c|c|}
\hline CT characteristic & $\begin{array}{l}\text { Whole cohort } \\
(n=24)\end{array}$ & $\begin{array}{c}\text { Group-IST } \\
(n=6)\end{array}$ & $\begin{array}{c}\text { Group-PBST } \\
(n=18)\end{array}$ & p-value \\
\hline Tumor size (mm) & $27.7(24-40.8)$ & $32.3(22.4-52.7)$ & $26.2(24-40.3)$ & 0.689 \\
\hline CBD diameter $(\mathrm{mm})$ & $14.5(11.7-15.8)$ & $15.2(11.8-17)$ & $14(10-16)$ & 0.548 \\
\hline MPD diameter (mm) & $4.9(2.1-7.9)$ & $7.7(3.2-9.6)$ & $4.8(2-7)$ & 0.171 \\
\hline $\begin{array}{l}\text { Lesion morphology }(\mathrm{n}, \%) \\
\text { Nodular } \\
\text { Infiltrative }\end{array}$ & $\begin{array}{c}9(37.5) \\
15(62.5)\end{array}$ & $\begin{array}{l}5(83.3) \\
1(16.7)\end{array}$ & $\begin{array}{c}4(22.2) \\
14(77.8)\end{array}$ & 0.015 \\
\hline CBD cut-off $(n, \%)$ & $18(75)$ & $4(66.7)$ & $14(77.8)$ & 0.618 \\
\hline CBD dilatation (n, \%) & $22(91.7)$ & $6(100)$ & $16(88.9)$ & $>0.999$ \\
\hline MPD cut-off $(n, \%)$ & $16(66.7)$ & $5(83.3)$ & $11(61.1)$ & 0.621 \\
\hline MPD dilatation (n, \%) & $16(66.7)$ & $5(83.3)$ & $11(61.1)$ & 0.621 \\
\hline Groove involvement $(n, \%)$ & $11(45.8)$ & $2(33.3)$ & $9(50)$ & 0.649 \\
\hline PDA involvement $(n, \%)$ & $10(41.7)$ & $3(50)$ & $7(38.9)$ & 0.665 \\
\hline $\begin{array}{l}\text { Enhancement pattern }(n, \%) \\
\text { Hypo- } \\
\text { Hyper- }\end{array}$ & $\begin{array}{c}15(62.5) \\
9(37.5)\end{array}$ & $\begin{array}{l}4(66.7) \\
2(33.3)\end{array}$ & $\begin{array}{c}11(61.1) \\
7(38.9)\end{array}$ & $>0.999$ \\
\hline Lymphatic involvement (n, \%) & $6(25)$ & $1(16.7)$ & $5(27.8)$ & $>0.999$ \\
\hline Metastasis $(n, \%)$ & $3(12.5)$ & $1(16.7)$ & $2(11.1)$ & $>0.999$ \\
\hline Ascites (n, \%) & $1(4.2)$ & $0(0)$ & $1(5.6)$ & $>0.999$ \\
\hline Vascular involvement $(\mathrm{n}, \%)$ & $3(12.5)$ & $0(0)$ & $3(16.7)$ & 0.546 \\
\hline
\end{tabular}

The upper normal diameter limit was upgraded for $1 \mathrm{~mm}$ per every decade of life in elderly ( $>60$ years). MPD cut-off was defined as an abrupt and blunt termination of main pancreatic duct. MPD dilation (Figure 3B) was taken as an MPD maximum diameter exceeding $3 \mathrm{~mm}$. Groove involvement was taken as replacement of fatty tissues with soft tissue in the pancreatoduodenal groove. Pancreaticoduodenal artery (PDA) involvement was defined as the longitudinal axis of PDA being wrapped around by the tumoral mass more than $180^{\circ}$. Hypoenhancement was present when the attenuation of the mass was lower than healthy parts of pancreatic corpus or tail in late portal phase. Otherwise, the mass was noted to be hyperenhanced. Lymphatic involvement was present when the short axis of at least one regional lymph node exceeded $1 \mathrm{~cm}$. Tumor size was the diameter measured in the longest axis. Additional arterial involvement meant that the longitudinal axis of the superior mesenteric, coeliac or common hepatic arteries being wrapped around by the tumor more than $180^{\circ}$.

Statistical analysis was performed with SPSS 19.0. The normality was analysed by Shapiro-Wilk test. Descriptive statistics were reported as median with quartiles (25th-75th percentile). Categorical variables were reported as frequencies with percentages. Independent two-group comparisons of continuous variables were tested using Mann-Whitney Utest. Proportions were compared using Fisher's exact test. Multiple-variable logistic regression analysis model was constructed to predict the presence of PBST. The biologically relevant clinical variables (CA 19-9, T stage) along with potentially predictor $\mathrm{CT}$ parameters were included in the model. Interobserver agreement was assessed using Kappa statistics. Significance level was accepted at $p<0.05$ for all statistical analyses.

\section{RESULTS}

Overall, 24 patients including 15 (62.5\%) males and nine (37.5\%) females were enrolled. The median age was 67.5 (60.5-76.5) years. Histomorphologic and immunohistochemical analyses revealed six ( $25 \%$ ) cases with IST and 18 (75 $\%)$ cases with PBST. Overall, $18(75 \%)$ patients were in THigh stage (15 PBST, 3 IST). Lymph node involvement as assessed by CT was present in six (25\%) patients. Distant metastasis was present in three $(12.5 \%)$ cases, who were not operated and endoscopic sampling was performed. CT characteristics in the whole cohort is presented in Table I.

The median age [72.5 (69-81) versus $63(57.75-75.5)$ years, respectively, $p=0.204$ ] and gender [3 (50\%) versus 12 $(66.7 \%)$ males, respectively, $p=0.635]$ did not differ significantly between the IST and PBST groups. The prevalence of patients undergoing surgical resection was similar between the groups $[4(66.7 \%)$ versus $14(77.8 \%)$, respectively, $\mathrm{p}=0.618]$. The prevalence of T-High stage was similar in both groups [5 (83.3\%) versus $15(83.3 \%)$, respectively, $p>0.999]$.

The prevalence of all predefined CT characteristics was similar between the groups ( $p>0.05$ for all) except for lesion morphology (Table I). Nodular lesion morphology was more frequent in the IST subgroup as compared to PBST [5 $(83.3 \%)$ versus $4(22.2 \%)$, respectively, $p=0.015]$. As a result, infiltrative lesion morphology was more frequent in PBST group.

Multiple variable logistic regression analysis revealed that 
infiltrative lesion morphology was the only independent predictor of PBST [Odds ratio: $14.9,95 \% \mathrm{Cl}: 1.2-186$, $\mathrm{p}=0.036]$. The sensitivity, specificity, positive predictive value, negative predictive value and overall diagnostic accuracy for $\mathrm{CT}$-assessed infiltrative lesion morphology in predicting the PBST were, $77.8 \%(95 \% \mathrm{Cl}: 52.4-93.6)$, 83.3\% (95\% Cl: 35.9-99.6), 93.3\% (95\% Cl: 69.7-98.8), $55.6 \%$ (95\% Cl: $32.9-76.1$ ), and $79.2 \%$ (95\% Cl: 57.9-92.9), respectively. Kappa statistics for assessment of Iesion morphology demonstrated moderate agreement between two raters (Cohen's Kappa: 0.55, p=0.007).

\section{DISCUSSION}

The main finding of the current study is that among several CT characteristics, only the lesion morphology was able to predict the histopathological subtype of PDACs. The infiltrative morphology was associated with PBST; whereas, the nodular morphology predicted IST with an acceptable overall diagnostic accuracy. Other CT features did not predict the histopathological subtype of PDACs. This is the first study evaluating the role of $C T$ in predicting the immunohistological subtypes of PDACs of periampullary origin.

An infiltrative lesion morphology was previously reported to be more commonly observed in PBST of ampullary adenocarcinomas. In an MRI study by Chung et al. and CT study by Ivanovic et $\left.a\right|_{;} ^{13,14}$ infiltrative appearance was more commonly observed in PBST; whereas, all IST tumors yielded a nodular appearance. Similarly, this study showed 14 times higher odds for infiltrative morphology to predict PBST. On the other hand, not all PDACs with IST had a nodular lesion morphology in this study. One plausible cause that may explain this discordant finding may be the differences between the populations studied. Both previous studies included only those with ampullary adenocarcinomas; whereas, the authors studied PDACs of periampullary origin in current study.

The CBD dilatation with or without MPD dilatation is encountered in more than $80 \%$ of cases with pancreatic head PDAC. ${ }^{15}$ In current study, CBD was dilated in $91 \%$ of the whole cohort, which is in line with the literature. Moreover, CBD dilatation with or without MPD dilatation was reported to be more common in PBST versus IST of ampullary carcinomas. ${ }^{13}$ Others did not confirm this finding. ${ }^{14}$ However, the rate of CBD dilatation was similar between the PBST and IST groups in current study. This discordant finding may be attributable to the differences between the populations studied in terms of tumor location. For periampullary PDACs, PBST may not necessarily be associated with more frequent CBD dilatation as it is for ampullary tumors.

Pancreaticoduodenal groove infiltration and PDA involvement were more frequently seen in the PBST of ampullary carcinomas. ${ }^{14}$ However, the authors did not observe such a difference in this series. The authors believe, groove and PDA involvements do not solely relate to the histopathological subtype. While a PBST tumor with a small size may be free of groove and PDA involvement, a large IST may involve both. In current study, tumor size was not different between the PBST and IST groups, which might have contributed to the similarity of prevalence of PDA and groove involvements.

Previous studies consistently have shown that periampullary cancer patients with positive lymph node status and vascular invasion have poorer prognosis. ${ }^{16}$ Since the histological phenotype is a better prognosticator in patients with periampullary cancers, ${ }^{17}$ one may expect a higher frequency of lymphatic involvement and vascular involvement in those with PBST as compared to IST. However, the rates of lymphatic or vascular involvement did not differ between the two groups in this study. Hence, a poor prognosis in PBST appears unrelated to lymphatic or vascular involvement.

The PDAC of periampullary origin is more frequently seen as a hypodense lesion at contrast enhanced $\mathrm{CT}$ when compared to normal pancreatic parenchyma. ${ }^{1}$ However, PDAC less frequently demonstrate hyperenhancement. ${ }^{18}$ In the current study, hyperenhancement was seen in $37 \%$ of cases. The frequency of hyperenhancement did not differ between the PBST and IST groups. Hence, enhancement pattern was considered not to be associated with histological subtype of periampullary PDACs.

Tumor size has been reported as a prognosticator in both periampullary and ampullary cancers. ${ }^{19,20}$ In this study, PBST and IST had no significant difference in terms of tumor size. Hence, a link between tumor size and hisotological subtype cannot be justified. The lower survival rate with PBST in published reports is probably due to the aggressive behaviour of these tumors rather than a size effect.

The main strength of this study is inclusion of a group of patients with PDAC of periampullary origin, who were not previously represented in studies revealing their imaging features, since IST was once not considered a differentiation pattern for this group. Another power of current study is that histomorphological and immunohistochemical confirmation was used as reference standard in all cases. The major drawback of the current study is the small sample size from a single centre. The retrospective design may be considered a limitation, as well. No case of mixed IST and PBST was observed in this study and CT assessment of mixed cases remains controversial.

\section{CONCLUSION}

The only CT characteristic to discriminate the histologic subtypes was the lesion morphology in patients with periampullary PDAC. An infiltrative appearance is associated with PBST; whereas, nodular appearance more likely predicts IST. 
Whether this information may be extrapolated to cases with nonresectable tumors and no chance for biopsy in order to select the appropriate chemotherapeutic agent requires confirmation with prospective studies.

\section{ETHICAL APPROVAL:}

Ethical approval was obtained from the Institutional Ethics Committee before the conduct of the study (Approval No. 2018/0015).

\section{PATIENTS' CONSENT:}

Informed consent could not be obtained from patients because this was a retrospective work and most patients' contact information was not up to date; and some patients died before the conduct of the study (due to malignant disease).

\section{CONFLICT OF INTEREST:}

The authors declared no conflict of interest.

\section{AUTHORS' CONTRIBUTION:}

NG: Design and conception, data acquisition, analysis and interpretation, drafting, critical review and comment of manuscript, approval of the final version of the manuscript.

FB, OA: Conception, data acquisition, critical review and comment of manuscript, approval of the final version of the manuscript.

HH, GD, AK: Design and conception, data acquisition, critical review and comment of manuscript, approval of the final version of the manuscript.

\section{REFERENCES}

1. Al-Hawary MM, Kaza RK, Francis IR. Optimal ımaging modalities for the diagnosis and staging of periampullary masses. Surg Oncol Clin N Am 2016; 25(2):239-53. doi: 10.1016/j.soc.2015.12.001.

2. Wiltberger G, Krenzien F, Atanasov G, Hau HM, Schmelzle M, Bartels $M$, et al. Pancreaticoduodenectomy for periampullary cancer: Does the tumour entity influence perioperative morbidity and long-term outcome? Acta Chir Belg 2018; 118(6):341-7. doi: 10.1080/00015458.2017. 1385894.

3. Westgaard A, Tafjord S, Farstad IN, Cvancarova M, Eide TJ, Mathisen 0 , et al. Pancreatobiliary versus intestinal histologic type of differentiation is an independent prognostic factor in resected periampullary adeno-carcinoma. BMC Cancer 2008; 8:170. doi: 10.1186/1471- 2407-8-170.

4. Zimmermann C, Wolk S, Aust DE, Meier F, Saeger HD, Ehehalt $\mathrm{F}$, et al. The pathohistological subtype strongly predicts survival in patients with ampullary carcinoma. Sci Rep 2019; 9(1):12676. doi: 10.1038/s41598-019-49179-w.

5. Albores-Saavedra J, Schwartz AM, Batich K, Henson DE. Cancers of the ampulla of Vater: Demographics, morphology, and survival based on 5,625 cases from the SEER program. J Surg Oncol 2009; 100(7):598-605. doi: $10.1002 / j s 0.21374$.

6. Westgaard A, Pomianowska E, Clausen OP, Gladhaug IP. Intestinal-type and pancreatobiliary-type adenocarcinomas: How does ampullary carcinoma differ from other periam-pul- lary malignancies? Ann Surg Oncol 2013; 20(2):430-9. doi: 10.1245/s10434-012-2603-0.

7. Albores-Saavedra J, Simpson K, Dancer YJ, Hruban R. Intestinal type adenocarcinoma: A previously unrecognised histologic variant of ductal carcinoma of the pancreas. Ann Diagn Pathol 2007; 11(1):3-9. doi: 10.1016/j.anndiagpath. 2006.06.008.

8. Bronsert P, Kohler I, Werner M, Makowiec F, Kuesters S, Hoeppner J, et al. Intestinal-type of differentiation predicts favourable overall survival: Confirmatory clinicopatho-logical analysis of 198 periampullary adenocarcinomas of pancreatic, biliary, ampullary and duodenal origin. BMC Cancer 2013; 13:428. doi: 10.1186/1471-2407-13-428.

9. Kumari N, Prabha K, Singh RK, Baitha DK, Krishnani N. Intestinal and pancreatobiliary differentiation in periam-pullary carcinoma: The role of immunohistochemistry. Hum Pathol 2013; 44(10):2213-9. doi: 10.1016/j.humpath. 2013.05.003.

10. Bakshi N, Dhawan S, Nundy S, Rao S, Chopra P, Bhalla S. Role of immunohistochemistry in the subtyping of periampullary adenocarcinoma. Int J Surg Pathol 2019; 27(6):598-608. doi: 10.1177/1066896919837606.

11. Elbanna KY, Jang HJ, Kim TK. Imaging diagnosis and staging of pancreatic ductal adenocarcinoma: A comprehensive review. Insights Imaging 2020; 11(1):58. doi: 10.1186/ s13244- 020-00861-y.

12. Nikolaidis P, Hammond NA, Day K, Yaghmai V, Wood CG 3rd, Mosbach DS, et al. Imaging features of benign and malignant ampullary and periampullary lesions. Radiographics 2014; 34(3):624-41. doi: 10.1148/rg. 343125191.

13. Ivanovic AM, Alessandrino F, Maksimovic R, Micev M, Ostojic $\mathrm{S}$, Gore RM, et al. Pathologic subtypes of ampullary adenocarcinoma: Value of ampullary MDCT for noninvasive preoperative differentiation. AJR Am J Roentgenol 2017; 208(3): W71-8. doi: 10.2214/AJR.16.16723.

14. Chung YE, Kim MJ, Park MS, Choi JY, Kim H, Kim SK, et al. Differential features of pancreatobiliary- and intestinal-type ampullary carcinomas at MR imaging. Radiology 2010; 257(2):384-393. doi: 10.1148/radiol.10100200.

15. Frampas E, David A, Regenet N, Touchefeu Y, Meyer J, Morla O. Pancreatic carcinoma: Key-points from diagnosis to treatment. Diagn Interv Imaging 2016; 97(12):1207-23. doi: 10.1016/j.diii.2016.07.008.

16. Chandrasegaram MD, Gill AJ, Samra J, Price T, Chen J, Fawcett J, et al. Ampullary cancer of intestinal origin and duodenal cancer - A logical clinical and therapeutic subgroup in periampullary cancer. World J Gastrointest Oncol 2017; 9(10):407-15. doi: 10.4251/wjgo.v9.i10.407.

17. Williams JL, Chan CK, Toste PA, Elliott IA, Vasquez CR, Sunjaya DB, et al. Association of histopathologic phenotype of periampullary adenocarcinomas with survival. JAMA Surg 2017; 152(1):82-8. doi: 10.1001/jamasurg.2016.3466.

18. Jang SK, Kim JH, Joo I, Jeon JH, Shin KS, Han JK, et al. Differential diagnosis of pancreatic cancer from other solid tumours arising from the periampullary area on MDCT. Eur Radiol 2015; 25(10):2880-8. doi: 10.1007/s00330015-3721-4.

19. El Nakeeb A, El Sorogy M, Ezzat H, Said R, El Dosoky M, Abd El Gawad $M$, et al. Predictors of long-term survival after 
pancreaticoduodenectomy for peri-ampullary adenocarcinoma: A retrospective study of 5-year survivors. Hepatobiliary Pancreat Dis Int 2018; 17(5):443-9. doi: 10.1016/ j.hbpd.2018.08.004.
20. Junrungsee S, Kittivarakul E, Ko-iam W, Lapisatepun W, Sandhu T, Chotirosniramit A. Prognostic factors and survival of patients with carcinoma of the ampulla of vater after pancreaticoduodenectomy. Asian Pac J Cancer Prev 2017; 18(1):225-9. doi: 10.22034/APJCP.2017.18.1.225. 